\title{
The Association Between Oral Contraceptives, Depot- Medroxyprogesterone Acetate, and Trichomoniasis
}

\author{
Michelle R. Torok, PHD ${ }^{\star}$, William C. Miller, MD, PHD, Marcia M. Hobbs, PHD ${ }^{\dagger}$, Pia D. M. \\ MacDonald, PHD ${ }^{\ddagger}$, Peter A. Leone, MD ${ }^{\dagger} \S$, Jane R. Schwebke, MDף, and Arlene C. Seña, \\ $\mathrm{MD}^{\dagger, \S}$ \\ "Department of Epidemiology, University of North Carolina at Chapel Hill (UNC) Gillings School of \\ Global Public Health, Chapel Hill, NC \\ tDepartment of Medicine, University of North Carolina at Chapel Hill, Chapel Hill, NC \\ ‡Department of Epidemiology, North Carolina Center for Public Health Preparedness, North \\ Carolina Institute for Public Health, University of North Carolina at Chapel Hill (UNC) Gillings \\ School of Global Public Health, Chapel Hill, NC \\ §Department of Infectious Diseases, University of North Carolina at Chapel Hill, Chapel Hill, NC \\ IDepartment of Medicine, University of Alabama at Birmingham, Birmingham, AL
}

\begin{abstract}
Background-Hormonal contraception use by women may increase the risk of acquiring certain sexually transmitted infections. We explored the effect of hormonal contraceptive use, specifically oral contraception (OC), and depot-medroxyprogesterone acetate (DMPA) on Trichomonas vaginalis infections in women.

Methods-We examined data from a prospective case-control study of women with trichomoniasis and noninfected female patients recruited from 3 public sexually transmitted disease clinics. Women with positive wet mount microscopy or $T$. vaginalis culture results were classified as having trichomoniasis. Participants underwent physical examinations, sexually transmitted infections testing and completed questionnaires which included information about demographics, sexual behavior, douching and contraceptive use. We assessed the association between hormonal contraceptives and trichomoniasis using bivariable and multivariable analysis and estimated exposure odds ratios (ORs) and adjusted odds ratios (aORs) with 95\% confidence intervals (CIs).
\end{abstract}

Results-We identified 427 women with trichomoniasis and 144 uninfected women who had information reported about contraception use. Compared with nonhormonal contraceptive use, OC use was negatively associated with trichomoniasis in bivariable analysis (OR: 0.5 ; 95\% CI: $0.3-$ 0.8 ). This association was no longer statistically significant after adjusting for demographic variables, douching and condom use (aOR: 0.9; 95\% CI: 0.5-1.6). Use of DMPA, compared with nonhormonal contraceptive use, was not associated with trichomoniasis in bivariable or multivariable analyses (OR: 1.0, 95\% CI: 0.5-2.1; aOR $=1.4,95 \%$ CI: 0.6-3.4, respectively).

Conclusions-Although OC use appeared to have a protective effect in the bivariable analysis, the hormonal contraceptives OC and DMPA were not associated with $T$. vaginalis infection after adjustment for other factors.

Copyright () 2009 American Sexually Transmitted Diseases Association All rights reserved.

Correspondence: Michelle Torok, PhD, 54A Plymouth Street, 4500, Wanganui, New Zealand. torok@email.unc.edu. 
Trichomonas vaginalis $(T V)$ is associated with women's health problems that include vaginitis, adverse pregnancy outcomes, cervical cancer and increased HIV transmission. ${ }^{1-5}$ The possible effect of hormonal contraceptive use on $T_{V}$ is of particular interest because over 100 million women worldwide use hormonal contraception such as oral contraceptives (OC), intrauterine devices (IUD), Norplant, and depot-medroxyprogest-erone acetate (DMPA). Sex hormones influence the reproductive immune response, 6,7 and hormonal contraceptive use affects the acquisition of other reproductive tract conditions, such as chlamydia, gonorrhea, candidiasis, and bacterial vaginosis (BV). ${ }^{7-18}$ Results from previous studies on hormonal contraceptive use and $T V$ are conflicting. ${ }^{7,9,18-28}$ This could be due, in part, to small sample sizes as well as differences in diagnostic and analytic methods used among studies. The objective of this case-control study was to determine the relationship between 2 types of hormonal contraceptive use, OC and DMPA, and prevalent $T_{V}$ in women attending sexually transmitted disease (STD) clinics in the Southeast United States.

\section{MATERIALS AND METHODS}

\section{Study Design and Sample}

We conducted a prospective, case-control study in 3 health department STD clinics located in Durham and Raleigh, NC and Birmingham, AL, from November 2001 to July 2003. The study was approved by the University of North Carolina at Chapel Hill and the University of Alabama at Birmingham Biomedical Institutional Review Boards.

Female patients who were English-speaking, $\geq 18$ years of age, and had no history of previous prescreening or study enrollment were prescreened for the study, which involved collection of specimens for wet mount microscopy and cultures for $T V$ detection. Women were eligible for study enrollment if they had not used metronidazole for 4 weeks before, and had a history of vaginal sex within 60 days of $T V$ culture collection. Women who had a positive $T_{V}$ wet mount microscopy test on prescreening received treatment and/or counseling and were immediately recruited into the study. Women who had a negative $T_{V}$ wet mount test on prescreening but positive $T V$ culture results were contacted to return to the clinic for treatment and/or counseling. These women were recruited into the study at their return visit.

For every 3 female subjects with positive wet mount microscopy results for $T V$ who were enrolled in the study, we recruited 1 prescreened woman with a negative wet mount microscopy result. This case-control ratio was selected because this design was part of a larger study that focused on enrolling women with $T_{V}$ infection and their male sexual partners. ${ }^{29}$ The cases and controls were not matched. Patients who were enrolled as controls but were subsequently diagnosed with trichomoniasis by culture were reclassified as cases. All enrolled participants provided written informed consent.

All study participants reported having had sex partners within 2 months before study enrollment. Women were asked whether they used different types of contraception (condom, spermicide, diaphragm, withdrawal, IUD, OC, DMPA, Nor-plant, had a hysterectomy or tubal ligation, partner had a vasectomy, or "other" type of birth control) while having vaginal sex with their sex partners within the past 2 months.

\section{Clinical, Demographic, and Behavioral Data}

A routine bimanual pelvic exam, vaginal specimen collection for wet mount microscopy, and testing for other STIs were performed on all women presenting to the clinics for evaluation. Routine testing for Chlamydia trachomatis was also performed, but was limited to women aged $\_5$ years in 1 clinic. HIV testing was offered to all prescreened women. In addition to routine specimen collection, vaginal swabs for $T_{V}$ culture, and Gram stains for 
BV diagnosis were collected from prescreened women. Upon enrollment, information about demographics (including age, race/ethnicity, income, and educational level), sexual behavior and douching practices was collected. Clinical information such as history of sexually transmitted infections (STIs) was also obtained. Demographic, behavioral, and clinical data were acquired from an in-person questionnaire administered by research personnel and from medical records.

\section{Laboratory Analyses}

Wet mount microscopy for $T V$ was performed according to routine clinic procedures by trained laboratory personnel at each site. Vaginal swabs were immediately placed in saline, agitated and 1 drop of the solution was observed at 200× magnification. T. vaginalis culture was performed using the InPouch culture system (Biomed, White City, OR). Culture swabs were inoculated into culture pouches and were read daily up to 5 days after inoculation. Testing for other STIs were also performed as previously described. ${ }^{29}$ Vaginal smear Gram stains were performed and evaluated at University of Alabama at Birmingham using Nugent's criteria. ${ }^{30}$

\section{Measures}

Women with positive wet mount microscopy and/or $T_{V}$ culture results (defined as the presence of motile trichomonads) were categorized as having $T V$. Women with negative wet mount microscopy results and negative $T_{V}$ culture results (defined as the absence of motile trichomonads) were categorized as not being infected with $T V$.

Hormonal contraception was defined to include oral contraception or birth control pills, DMPA, and Norplant (levonorgestrel). Nonhormonal contraceptives included the diaphragm, hysterectomy, partner vasectomy, withdrawal and spermicide. IUDs could be categorized as either hormonal if they contained levonorgestrel or nonhormonal for the inert, copper-based devices. However, because the frequency of observations were small for Norplant $(n=7)$ and IUDs $(n=6)$, these contraceptives were not included in bivariable or multivariable analyses.

A 3-level nominal exposure variable was created to assess hormonal contraceptive use among the women with their most recent and/or most frequent sexual partners. ${ }^{29}$ The first category consisted of women who used OC, the second category comprised women who used DMPA, and the last category was made up of women who used nonhormonal contraception with their most recent and/or most frequent sexual partners. Condom use was not included in the exposure variable, because most women do not exclusively use condoms. In this study, $45 \%$ of the women reported condom use, but only $9 \%$ of the women reported exclusive condom use. However, since condom use can impact $T V$ transmission, whether the participant used condoms with their sexual partner in the past 2 months was included in bivariable analyses to assess potential confounding. In the analyses, each of the 2 exposed groups (women who used OC and women who used DMPA) was compared with the unexposed group, defined as women who used nonhormonal contraception.

\section{Statistical Analyses}

Data were double entered and analyzed using SAS software version 8 (SAS Corporation, Cary, NC). Univariable analyses were conducted using frequency distributions of categorical variables. Measures of central tendency and variability were assessed for continuous variables. Bivariable analyses were performed to assess the association of selected demographic and behavioral variables with trichomoniasis. 
Demographics (age, race/ethnicity, last year's household income, highest educational level attained), sexual behavior (past diagnosis with an STI other than $T V$, coinfection with gonorrhea, condom use in the past 2 months, number of different sex partners in the past year, number of sexual partners in the past 2 months and number of times the woman had sex in an average week), douching behavior (number of times in the past week, number of times in an average week), and factors related to the vaginal microenvironment (concurrent diagnosis with $\mathrm{BV}$, level of vaginal lactobacilli) were assessed for inter-subgroup differences and for confounding the associations between hormonal contraceptive use and $T V$.

We initially tested each covariable for intersubgroup differences and confounding by creating a model with the exposure variable and 1 additional covariable, and comparing it with a model containing only the exposure variable. Variables with substantive differences in the exposure odds ratio (OR) and a likelihood ratio (LR) $P$ value $\$ 0.10$ were assessed in multivariable analyses for intersubgroup differences. Variables that resulted in at least a $10 \%$ change in the OR were evaluated in multivariable models for confounding.

Adjusted prevalence OR and 95\% CI for the association of interest were estimated using unconditional multiple logistic regression. We used a backward elimination strategy. Intersub-group differences were evaluated by comparing the LR for the models with and without the interaction term and retaining variables with a LR $P$ value $\$) .10$. Confounding was assessed by comparing the fully adjusted OR with the adjusted OR after removing potentially confounding variables in order of the magnitude of the Wald $X^{2}$ test $P$ value. We repeated this process for covariables until the cumulative change in the OR for the exposure was $>10 \%$ compared with the fully adjusted OR.

Since $T_{V}$ testing is limited in most clinics, we did not include history of previous $T_{V}$ infection in our analyses. However, we performed a sensitivity analysis to evaluate the influence of prior $T V$ infection on the outcome by adjusting our final models for this variable and assessing its influence on our results.

\section{RESULTS}

\section{Study Population}

Seven hundred ninety women were diagnosed with $T_{V}$ by positive wet mount and/or culture during prescreening, and 539 (68\%) infected women participated in the study. Of these, 347 women reported nonhormonal contraceptive use, 50 reported using OC, and 30 women used DMPA; these 427 women (79\%) were included in the current analyses. Of the 203 women negative for $T_{V}$ recruited for the study, 177 (87\%) agreed to participate. Of these, 144 (81\%) female controls were included in these analyses, of which 103 reported using non-hormonal contraceptives, 31 used OC, and 10 used DMPA.

Among the 571 female participants with reported contraceptive use, the median age was 29.6 years (standard deviation, 9.0 years). Most women reported that they were black (92.3\%) in race/ethnicity, and $64.1 \%$ had a high school level of education or less. Last year's household income was reported as less than $\$ 12,000$ for half of the women in the study. The majority (77.2\%) of women had previously been diagnosed with an STI other than $T V$, and 66.0\% had more than 1 sex partner in the past year. Most women (85.8\%) did not use OC, and $54.2 \%$ reported using condoms in the past 2 months.

\section{Characteristics Associated With T. vaginalis}

Older women (age 237 years) were more likely to have prevalent trichomoniasis compared with younger women (Table 1). Black women were more likely to be infected with $T V$ 
compared with nonblack women, as were women with less (vs. more) education. Age, race, and education remained significantly associated with trichomoniasis in the multivariable analysis. Having had a past STI diagnosis, having had more than 1 (vs. 1) sexual partner in the past year, BV status, and household income were not associated with having trichomoniasis (Table 1).

\section{Association Between Hormonal Contraceptive Use and T. vaginalis}

In bivariable analyses, we observed an inverse association between OC use compared with nonhormonal contraceptive use, and prevalent $T V$ (OR: 0.5 ; 95\% CI: $0.3-0.8$ ). After adjusting for age, race/ethnicity, highest educational level attained, income, number of times the woman douched in an average week, and condom use, this association no longer reached statistical significance (OR: 0.9; 95\% CI: 0.5-1.6).

There was no association between women who used DMPA and $T_{V}$ in bivariable analysis (OR: 1.0; 95\% CI: 0.5-2.1). Adjusting for age, race/ethnicity, education, income, number of times the woman douched in an average week, and condom use did not appreciably change these results (OR: 1.4; 95\% CI: 0.6-3.4) (Table 1).

Since routine $T_{V}$ testing is limited in most STD clinics, we did not include prior $T_{V}$ diagnosis in our analyses but added it to our final model to assess its effect on our results. Addition of prior $T_{V}$ diagnosis did not substantively change our results ( $\mathrm{OC}$ use: $\mathrm{OR}=0.9$, $95 \% \mathrm{CI}=0.5-1.7$; DMPA use: $\mathrm{OR}=1.4,95 \% \mathrm{CI}=0.6-3.6$ ).

\section{DISCUSSION}

Oral contraceptive use was marginally protective against $T V$ in our bivariable analysis. After adjusting for covariables, however, this association was not statistically significant. Our results are consistent with other investigators who reported no association between OC and $T_{V}, 7,9,11,14,19,24,31,32$ although direct comparison of results is challenging due to differences in diagnostic and analytical methods. Most studies reporting a statistically significant protective effect of OC on $T V$ used wet mount microscopy for diagnosis, bivariable analyses, or failed to adjust for potentially important confounders such as age. . $^{215,20,22,23,25}$ To our knowledge, this study is the first to use both $T_{V}$ culture as the diagnostic reference standard and to evaluate potentially important covariables in multivariable analyses.

Although we found no multivariable association between OC or DMPA and trichomoniasis, sex hormones are known to affect STIs. ${ }^{6}$ Oral contraceptives are thought to influence vaginal conditions and susceptibility to infection, including chlamydia, vaginal candidiasis, and BV. 7,9,13,14,22,25,33 The impact of OC use on $T_{V}$ has not been determined although several mechanisms for protection by OC against trichomoniasis have been hypothesized. Oral contraceptives might alter host defenses to $T V$ because female sex hormones are known to affect the reproductive tract immune response. ${ }^{34-37}$ For example, $T_{V}$ infects vaginal epithelium, which may be thicker in women using OC compared with women not on hormonal contraception. ${ }^{38}$ The effects of OC hormones on the vaginal epithelium may create an environment that is less hospitable for trichomonads. OC might also protect against trichomoniasis by lowering vaginal lactoferrin concentrations. The mean vaginal lactoferrin concentration in women on $\mathrm{OC}$ is significantly lower compared with women not on OC, and is the primary source of iron for trichomonads. ${ }^{39}$ In vitro, $T_{V}$ is positively regulated by iron resulting in increased cytoadherance to vaginal epithelium, resistance to complement lysis and synthesis of trichomonad adhesions. ${ }^{40-42}$ Therefore, $T V$ may be less virulent in women taking OC. 
Estrogen may also directly affect $T V$, which possesses estrogen receptors, and have been demonstrated to slow $T V$ growth and decrease its attachment to mammalian cells when added to cultures. ${ }^{43-45}$ However, in vivo studies suggest that estrogen may promote growth of $T V$. For example, the persistence of trichomoniasis was reported in a postmenopausal woman (who was allergic to metronidazole) until she discontinued estrogen replacement therapy. ${ }^{46}$ If estrogen promotes $T_{V}$ growth in vivo, we might expect to observe a positive association between OC and $T v$. However, we found a possible inverse association between $\mathrm{OC}$ and $T V$ in bivariable analysis that may be due to a net reduction of systemic and/or local estrogen in women from OC. OC users are thought to have comparatively lower serum levels of endogenous estrogen than women who do not use hormonal contraceptives. ${ }^{47}$ The observed protective effect of $\mathrm{OC}$ could also result from the competitive binding of exogenous estrogen to $T_{V}$ estrogen receptors, ${ }^{22}$ impeding the stimulating effect of endogenous estrogen. However, this association was no longer statistically significant in multivariable analyses.

We found that DMPA use is not associated with having trichomoniasis. Few other studies have evaluated this relationship, although authors of 2 studies conducted in Africa also reported no association between DMPA and trichomoniasis compared with women using no contraception. ${ }^{26,32}$ Both of these studies in Africa diagnosed $T V$ using wet mount microscopy. Although the reason for a potential effect of DMPA use on $T_{V}$ is not known, some of the explanations discussed above, such as hormonal influence on the immune system, may be biologically plausible.

The limitations of our study merit discussion. We did not collect information regarding the duration of contraceptive use. Thus, it is possible that $T_{V}$ infection occurred before contraceptive use, resulting in exposure misclassification. We also lacked information on hormonal contraceptive composition. Most OC include a combination of estrogen and progesterone, though progesterone-only prescriptions exist. Furthermore, levels of progesterone and estrogen may differ among combination OC. However, OC misclassification should be nondifferential about $T_{V}$ status and any resulting bias should be toward the null. ${ }^{48}$ Trichomoniasis may also have been misclassified because of the imperfect performance of culture detection. Although it is considered the diagnostic reference standard, $T V$ culture is less sensitive than newer $T V$ nucleic acid amplification based tests. Thus, some $T_{V}$ diagnoses may have been missed. Likewise, this misclassification should be nondifferential about contraceptive status, and the resulting bias should lead to an underestimation of the true association.

Millions of women worldwide use contraception, and knowledge that contraceptives impact STIs, may affect a woman's contraceptive choice. We found no association between OC and $T_{V}$ nor between DMPA and $T V$ compared with women who use nonhormonal methods of contraception. Given the popularity of hormonal contraception, the high burden of $T_{V}$ in certain populations and its health consequences, confirmation of these results from studies which accurately and prospectively measure hormonal contraceptive use and assess $T_{V}$ infections using nucleic acid amplification based tests would be beneficial.

\section{Acknowledgments}

Supported by the National Institutes of Health STD Clinical Trials Unit grant N01AI075329, and the NC STI/TM Cooperative Research Center grant U19AI031496.

\section{References}

1. WHO. Global prevalence and incidence of selected curable sexually transmitted infections. Geneva, Switzerland: WHO; 2001. 
2. Cotch M, Pastorek J, Nugent R, et al. Demographic and behavioral predictors of Trichomonas vaginalis infection among pregnant women. Obstet Gynecol. 1991; 78:1087-1092. [PubMed: 1945213]

3. Laga M, Manoka A, Kivuvu M, et al. Non-ulcerative sexually transmitted diseases as risk factors for HIV-1 transmission in women: Results from a cohort study. AIDS. 1993; 7:95-102. [PubMed: 8442924]

4. Soper D. Trichomoniasis: Under control or undercontrolled? Am J Obstet Gynecol. 2004; 190:281290. [PubMed: 14749674]

5. Sorvillo F, Smith L, Kerndt P, et al. Trichomonas vaginalis, HIV, and African-Americans. Emerg Infect Dis. 2001; 7:927-932. [PubMed: 11747718]

6. Brabin L. Hormonal markers of susceptibility to sexually transmitted infections: Are we taking them seriously? BMJ. 2001; 323:394-395. [PubMed: 11509437]

7. Baeten JM, Nyange PM, Richardson BA, et al. Hormonal contraception and risk of sexually transmitted disease acquisition: Results from a prospective study. Am J Obstet Gynecol. 2001; 185:380-385. [PubMed: 11518896]

8. Jacobson DL, Peralta L, Farmer M, et al. Relationship of hormonal contraception and cervical ectopy as measured by computerized planimetry to chlamydial infection in adolescents. Sex Transm Dis. 2000; 27:313-319. [PubMed: 10907905]

9. Ceruti M, Canestrelli M, Condemi V, et al. Methods of contraception and rates of genital infections. Clin Exp Obstet Gynecol. 1994; 21:119-123. [PubMed: 8070115]

10. United Nations. World Contraceptive Use 2005. 2005; 2006

11. Avonts D, Sercu M, Heyerick P, et al. Incidence of uncomplicated genital infections in women using oral contraception or an intra-uterine device: A prospective study. Sex Transm Dis. 1990; 17:23-29. [PubMed: 2305333]

12. Moi H. Prevalence of bacterial vaginosis and its association with genital infections, inflammation, and contraceptive methods in women attending sexually transmitted disease and primary health clinics. Int J STD AIDS. 1990; 1:86-94. [PubMed: 1965491]

13. McGregor J, Hammill H. Contraception and sexually transmitted diseases: Interactions and opportunities. Am J Obstet Gynecol. 1993; 168:2033-2041. [PubMed: 8512049]

14. Oh MK, Feinstein RA, Soileau EJ, et al. Chlamydia trachomatis cervical infection and oral contraceptive use among adolescent girls. J Adolesc Health Care. 1989; 10:376-381. [PubMed: 2808080]

15. Gertig DM, Kapiga SH, Shao JF, et al. Risk factors for sexually transmitted diseases among women attending family planning clinics in Dar-es-Salaam, Tanzania. Genitourin Med. 1997; 73:39-43. [PubMed: 9155554]

16. Sonnex C. Influence of ovarian hormones on urogenital infection. Sex Transm Inf. 1998; 74:1119.

17. Morrison CS, Bright P, Wong EL, et al. Hormonal contraceptive use, cervical ectopy, and the acquisition of cervical infections. Sex Transm Dis. 2004; 31:561-567. [PubMed: 15480119]

18. Mohllajee AP, Curtis KM, Martins SL, et al. Hormonal contraceptive use and risk of sexually transmitted infections: A systematic review. Contraception. 2006; 73:154-165. [PubMed: 16413846]

19. Lefevre JC, Averous S, Bauriaud R, et al. Lower genital tract infections in women: Comparison of clinical and epidemiologic findings with microbiology. Sex Transm Dis. 1988; 15:110-113. [PubMed: 3135608]

20. Bramley M, Kinghorn G. Do oral contraceptives inhibit Trichomonas vaginalis? Sex Transm Dis. 1979; 6:261-263. [PubMed: 316933]

21. Fouts AC, Kraus SJ. Trichomonas vaginalis: Reevaluation of its clinical presentation and laboratory diagnosis. J Infect Dis. 1980; 141:137-143. [PubMed: 6965976]

22. Barbone F, Austin H, Louv WC, et al. A follow-up study of methods of contraception, sexual activity, and rates of trichomoniasis, candidiasis, and bacterial vaginosis. Am J Obstet Gynecol. 1990; 163:510-514. [PubMed: 2167008] 
23. Pereira LH, Embil JA, Haase DA, et al. Cytomegalovirus infection among women attending a sexually transmitted disease clinic: Association with clinical symptoms and other sexually transmitted diseases. Am J Epidemiol. 1990; 131:683-692. [PubMed: 2156421]

24. Staerfelt F, Gundersen TJ, Halsos AM, et al. A survey of genital infections in patients attending a clinic for sexually transmitted diseases. Scand J Infect Dis Suppl. 1983; 40:53-57. [PubMed: 6607522]

25. Kinghorn GR, Waugh MA. Oral contraceptive use and prevalence of infection with Chlamydia trachomatis in women. Br J Vener Dis. 1981; 57:187-190. [PubMed: 7237082]

26. Nsofor BI, Bello CS, Ekwempu CC. Sexually transmitted disease among women attending a family planning clinic in Zaria, Nigeria. Int J Gynaecol Obstet. 1989; 28:365-367. [PubMed: 2565260]

27. Lavreys L, Chohan V, Overbaugh J, et al. Hormonal contraception and risk of cervical infections among HIV-1-seropositive Kenyan women. AIDS. 2004; 18:2179-2184. [PubMed: 15577651]

28. Kazerooni T, Mosalaee A. Does contraceptive method change the Pap smear finding? Contraception. 2002; 66:243-246. [PubMed: 12413619]

29. Sena AC, Miller WC, Hobbs MM, et al. Trichomonas vaginalis infection in male sexual partners: Implications for diagnosis, treatment, and prevention. Clin Infect Dis. 2007; 44:13-22. [PubMed: 17143809]

30. Nugent RP, Krohn MA, Hillier SL. Reliability of diagnosing bacterial vaginosis is improved by a standardized method of gram stain interpretation. J Clin Microbiol. 1991; 29:297-301. [PubMed: 1706728]

31. Reed BD, Huck W, Zazove P. Differentiation of Gardnerella vaginalis, Candida albicans, and Trichomonas vaginalis infections of the vagina. J Fam Pract. 1989; 28:673-680. [PubMed: 2656903]

32. McClelland RS, Sangare L, Hassan WM, et al. Infection with Trichomonas vaginalis increases the risk of HIV-1 acquisition. J Infect Dis. 2007; 195:698-702. [PubMed: 17262712]

33. Cottingham J, Hunter D. Chlamydia trachomatis and oral contraceptive use: A quantitative review. Genitourin Med. 1992; 68:209-216. [PubMed: 1398654]

34. Prakash M, Patterson S, Gotch F, et al. Ex vivo analysis of HIV-1 co-receptors at the endocervical mucosa of women using oral contraceptives. BJOG. 2004; 111:1468-1470. [PubMed: 15663139]

35. Beagley KW, Gockel CM. Regulation of innate and adaptive immunity by the female sex hormones oestradiol and progester-one. FEMS Immunol Med Microbiol. 2003; 38:13-22. [PubMed: 12900050]

36. White HD, Crassi KM, Givan AL, et al. CD3+ CD8+ CTL activity within the human female reproductive tract: Influence of stage of the menstrual cycle and menopause. J Immunol. 1997; 158:3017-3027. [PubMed: 9058841]

37. Ghanem KG, Shah N, Klein RS, et al. Influence of sex hormones, HIV status, and concomitant sexually transmitted infection on cervicovaginal inflammation. J Infect Dis. 2005; 191:358-366. [PubMed: 15633094]

38. Ildgruben AK, Sjoberg IM, Hammarstrom ML. Influence of hormonal contraceptives on the immune cells and thickness of human vaginal epithelium. Obstet Gynecol. 2003; 102:571-582. [PubMed: 12962945]

39. Cohen MS, Britigan BE, French M, et al. Preliminary observations on lactoferrin secretion in human vaginal mucus: Variation during the menstrual cycle, evidence of hormonal regulation, and implications for infection with Neisseria gonorrhoeae. Am J Obstet Gynecol. 1987; 157:11221125. [PubMed: 3120589]

40. Ryu J, Choi H, Min D, et al. Effect of iron on the virulence of Trichomonas vaginalis. J Parasitol. 2001; 87:457-460. [PubMed: 11318588]

41. Alderete J, Provenzano D, Lehker M. Iron mediates Trichomonas vaginalis resistance to complement lysis. Microb Pathog. 1995; 19:93-103. [PubMed: 8577239]

42. Lehker M, Arroyo R, Alderete J. The Regulation by iron of the synthesis of adhesins and cytoadherence levels in the protozoan Trichomonas vaginalis. J Exp Med. 1991; 174:311-318. [PubMed: 1856625] 
43. Sugarman B, Mummaw N. Oestrogen binding by and effect of estrogen on trichomonads and bacteria. J Med Microbiol. 1990; 32:227-232. [PubMed: 2391707]

44. Ford LC, Hammill HA, DeLange RJ, et al. Determination of estrogen and androgen receptors in Trichomonas vaginalis and the effects of antihormones. Am J Obstet Gynecol. 1987; 156:1119_ 1121. [PubMed: 3495180]

45. Sugarman B, Mummaw N. The effect of hormones on Trichomonas vaginalis. J Gen Microbiol. 1988; 134:1623-1628. [PubMed: 3265429]

46. Sharma R, Pickering J, Mccormack W. Trichomoniasis in a postmenopausal women cured after discontinuation of estrogen replacement therapy. Sex Transm Dis. 1997; 24:543-545. [PubMed: 9339974]

47. Ildgruben A, Sjoberg I, Hammarstrom ML, et al. Steroid receptor expression in vaginal epithelium of healthy fertile women and influences of hormonal contraceptive usage. Contraception. 2005; 72:383-392. [PubMed: 16246667]

48. Rothman, K.; Greenland, S. Modern Epidemiology. 2. Philadelphia, PA: Lippincott Williams \& Wilkins; 1998.

49. Paavonen J. Physiology and ecology of the vagina. Scand J Infect Dis Suppl. 1983; 40:31-35. [PubMed: 6582587] 


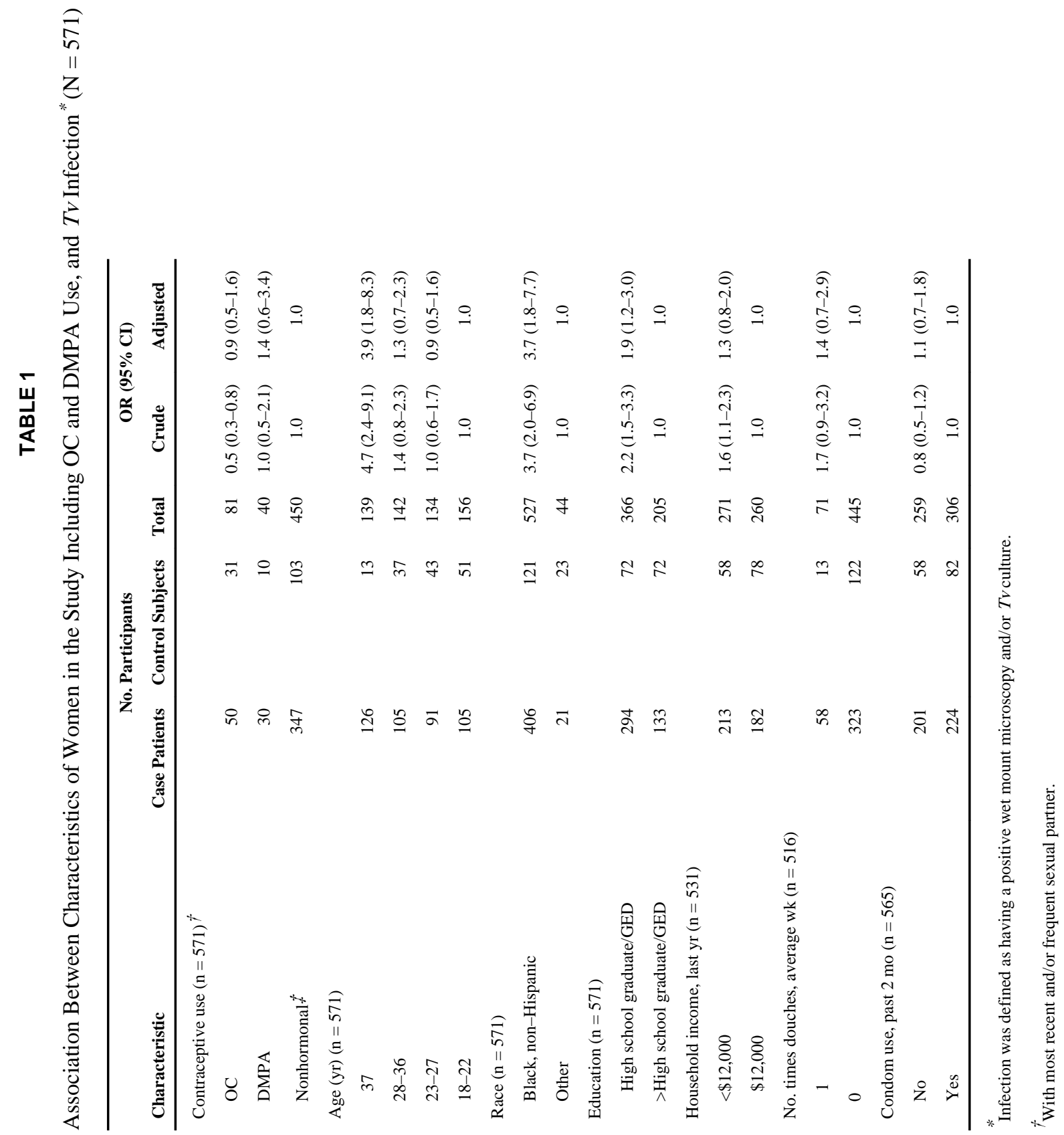


